\title{
Local Collection Efficiency in the Nano-Crystalline Solar Cells
}

Mohammad Javadi, Yaser Abdi ${ }^{*}$ and Ezatollah Arzi

Nanophysics Research Laboratory, Department of Physics, University of Tehran, Kargar Street, Tehran, Iran.

* Corresponding author. Tel/Fax: +98 21 61118610, E-mail address: y.abdi@ut.ac.ir (Y. Abdi). 


\begin{abstract}
A geometrical model based on the stochastic nature of diffusion transport is proposed to formulate local collection efficiency in the nano-crystalline solar cells. It is shown that this parameter is given by $\eta_{\text {local }}(x) \approx 1-x / L$. Using this formula, we derived the generalized form of the well-known equation of total collection efficiency $\left(\eta_{\text {coll }}=1-\tau_{I M P S} / \tau_{I M V S}\right)$, which can be used for all solar cells independent of their specific performance. The generalized equation is in good agreement with the simulation and empirical results reported by other groups. For more justification of the model validity, the results are compared with the non-linear diffusion equation. In addition, we propose an approach for direct estimation of the electron diffusion length based on the IMPS and IMVS responses.
\end{abstract}




\section{Introduction}

The new generation of solar cells are promising candidates for secure and clean energy harvesting. A typical nano-crystalline solar cell includes a porous nanostructured semiconductor $\left(\mathrm{TiO}_{2}, \mathrm{ZnO}, \mathrm{WO}_{3}\right)$ [1-4], a single layer of sensitizer (dye, quantum dots, perovskite)[5-8], and a hole transport medium (electrolyte containing $I^{-} / I_{3}^{-}$redox couple, HTM) $[9,10]$ filling the pores of semiconductor. Incident light is absorbed by sensitizer layer, and the excited electron-hole pair is injected respectively to porous semiconductor and hole transport medium from which they can travel toward external circuit to complete the energy harvesting cycle. Since the porous medium is surrounded by hole transport material, the net macroscopic electrical field in the electron transport medium is zero and the electron transport is believed to be carried out by diffusion.[11]

Recombination of electrons with the acceptor species in the electrolyte-based solar cells, or other kinds of hole carriers in the similar devices, leads to a finite diffusion length $(L)$, which is defined as the average distance that an electron travels before disappearing.[12] The internal gain or total collection efficiency of a solar cell is critically related to the electron diffusion length.[13-18] Experimentally, there are several methods to measure total collection efficiency and electron diffusion length in nanostructured solar cells, including steady state spectral characterization[19-21], time transient photovoltage and photocurrent measurements[22-25], and frequency resolved measurements (i.e. intensity modulated photocurrent spectroscopy (IMPS), intensity modulated photovoltage spectroscopy (IMVS), and electrochemical impedance spectroscopy (EIS)). [26-32]

In the frequency resolved measurements (IMVS and IMPS), the total collection efficiency $\left(\eta_{\text {coll }}\right)$ is written as a function of experimentally derived time constants as [26, 27, 33, 34]

$$
\eta_{\text {coll }}=\frac{\int_{0}^{d} G(x) \eta_{\text {local }}(x) d x}{\int_{0}^{d} G(x) d x}=f\left(\tau_{\text {IMPS }}, \tau_{\text {rec }}\right)
$$

where $G(x)$ is the electron generation rate and $d$ is semiconductor network thickness. $\eta_{\text {local }}(x)$ is the local collection efficiency defined as the probability of collection of an electron injected to nanostructured network at distance $x$ from collecting electrode. The IMPS time constant $\left(\tau_{I M P S}\right)$ is the characteristic time of disappearance of the electrons from the semiconductor network due 
to either recombination or extraction[28], and the IMVS time constant $\left(\tau_{I M V S}\right)$ is interpreted as the electrons lifetime (for a brief discussion see appendix A). Among different models having been proposed to explain the results of these dynamical measurements, one of the most used models rewrites Eq.1 as [11, 28, 35]

$$
\frac{\tau_{I M V S}}{\tau_{\text {coll }}}=\frac{\eta_{\text {coll }}}{1-\eta_{\text {coll }}}
$$

where $\tau_{\text {coll }}$ is the collection characteristic time constant. Substituting $\tau_{\text {IMPS }}^{-1}=\tau_{I M V S}^{-1}+\tau_{\text {coll }}^{-1}$ into Eq.1, the well-known form of total collection efficiency can be deduced as $\eta_{\text {coll }}=1-$ $\tau_{I M P S} / \tau_{I M V S}$. Recently, Bertoluzzi and Ma argued that the phenomenological Eq.2 leads to the wrong collection efficiency especially for $d \leq L$.[36] This argument is based on the assumption of $\tau_{\text {IMPS }} \approx \mathrm{d}^{2} / \mathrm{D}$, which leads to $\eta_{\text {coll }} \approx 1-(d / \mathrm{L})^{2}$. It should be noted that the assumption of $\tau_{\text {IMPS }} \approx \mathrm{d}^{2} / \mathrm{D}=\tau_{\text {transport }}$ basically ignores the possibility of electron recombination. However, as mentioned before, $\tau_{I M P S}$ is governed by disappearance of the electrons from the semiconductor network due to either recombination or extraction. Therefore, the assumption of $\tau_{\text {IMPS }} \approx \mathrm{d}^{2} / \mathrm{D}$ could not produce accurate results. On the other hand, according to the experimental and simulation results, the collection efficiencies obtained by Eq.2 are acceptable only for high-performance dye-sensitized solar cells (DSSCs).[28, 35] In the current study, we propose a geometrical model to formulate local collection efficiency. Utilizing this model, we will explain why Eq.2 is applicable only for high-performance DSSCs, and then we introduce the generalized form of Eq. 2 which can be used for all solar cells independent of their specific performance.

\section{Theory}

\subsection{Geometrical model for local collection efficiency}

The rate of charge delivery to the external circuit depends on the competition between transport and recombination of the electrons. Let us consider the transport of an electron in an infinitely large transport medium. Also, assume that the spatial position of the electron is far from collecting electrode. The electron moves randomly in the medium, and due to the finite diffusion length, it will be recombined at distance $L$ from the initial position. If we repeat this 
mental experiment for many times (with a fixed initial position), it is observed that just before recombination, the electron would be located on the surface of a sphere with average radius $L$ centered at the initial position (Fig.1.(a)). We call this sphere "Recombination sphere (RS)". Given the stochastic nature of the diffusion transport, the electron can be situated at any point on the RS surface with the same probability. Now, in the case of finite medium and in the presence of the collecting electrode, the electron can be transferred to the external circuit if there is an overlap between its recombination sphere and the collecting electrode (Fig.1.(b)). Considering these arguments, we can write the local collection efficiency of an electron injected to the transport medium at distance $x$ from collecting electrode as $\eta_{\text {local }}(x) \propto \tilde{s} / S$, where $\tilde{s}$ is the portion of the RS surface which is placed behind the collecting plane and $S$ is the total area of RS surface. It is noted that if the RS does not cross the collecting plane, the electron cannot be transferred to the external circuit because an electron recombines at the surface of RS before reaching the collecting electrode. In this case $\eta_{\text {local }}(x)$ is zero. By using the schematics shown in Fig.1, the following relation can be obtained

$$
\frac{\tilde{S}}{S}=\frac{L^{2} \int_{0}^{2 \pi} d \varphi \int_{0}^{\theta} \sin (\theta) d \theta}{4 \pi L^{2}}=\frac{1-\cos \theta}{2}
$$

By replacing $\cos \theta=x / L$ into the Eq. 3 and using the boundary condition of $\eta_{\text {local }}(x=0)=1$ and $\eta_{\text {local }}(x=L)=0$ [37-39], the local collection efficiency is obtained as

$$
\eta_{\text {local }}(x)= \begin{cases}1-x / L ; x \leq L \\ 0 & ; x>L\end{cases}
$$

It should be noted that the diffusion length used in Eq.4 can be considered either as a local parameter (i.e. a position-dependent function $L=L(x)$ ) or a global parameter (i.e. constant through the cell). 

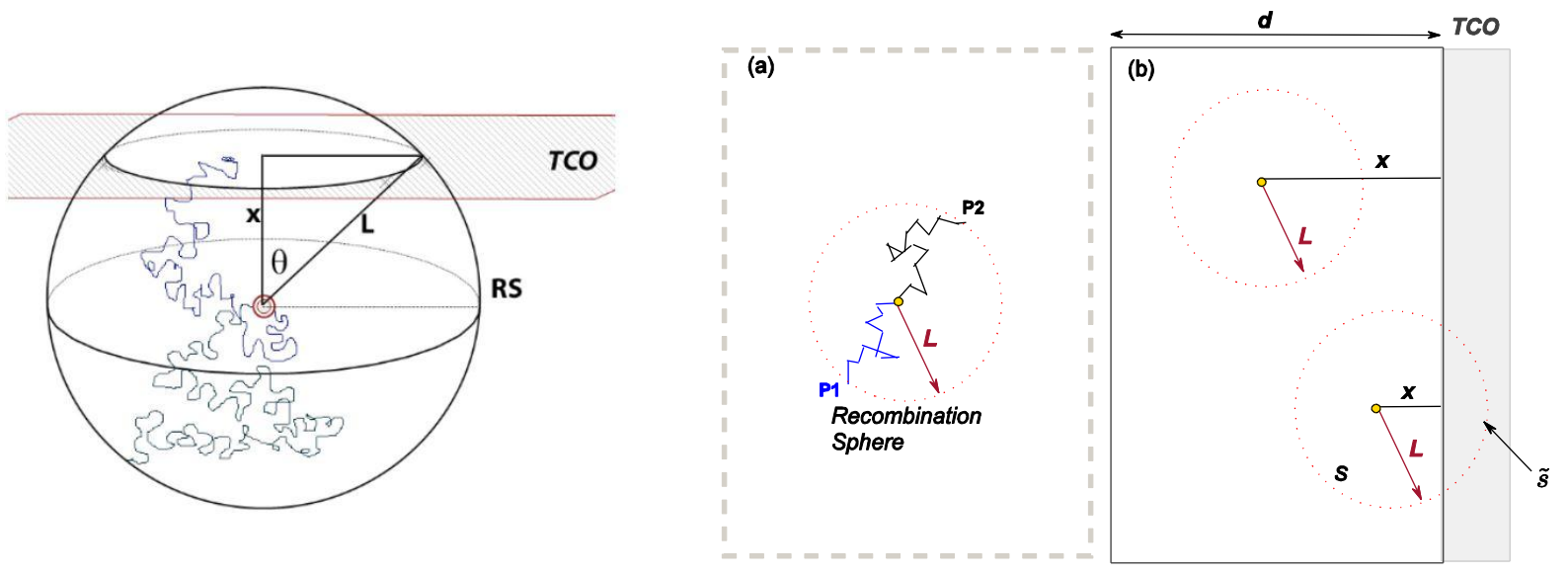

Figure.1 Left: A schematic of the recombination sphere (RS). The red circle represents a nanoparticle in the transport medium. The random blue and gray trajectories correspond respectively to the collection and recombination probabilities. The flat plane represents transparence collecting electrode (TCO). Right: (a) recombination sphere in an infinitely large medium. Yellow point indicates the initial position of the electron. Considering the stochastic nature of the diffusion transport, the electron can be situated at any point on the RS surface with the same probability $(P 1=P 2)$. (b) Recombination sphere in a finite medium in the presence of TCO. For $x>L$, the sphere does not cross TCO plane and $\eta_{\text {local }}(x)=0$ while for $x<L$, the RS overlap with TCO and the local collection efficiency is given by Eq.4.

\subsection{Generalized equation of the total collection efficiency}

Denoting $P_{\text {coll }}(x)\left(P_{\text {rec }}(x)\right)$ as the probability of collection (recombination), the local collection efficiency can be rewritten as

$$
\eta_{\text {local }}(x)=\frac{P_{\text {coll }}(x)}{P_{\text {coll }}(x)+P_{\text {rec }}(x)}=\frac{\tau_{\text {coll }}^{-1}(x)}{\tau_{\text {coll }}^{-1}(x)+\tau_{\text {IMVS }}^{-1}(x)}
$$

Here, $\tau_{\text {coll }}(x)$ is the time required for collection of an electron injected to the transport medium at distance $x$ from collecting electrode. After substituting Eq.4 into Eq.5, we obtain $\tau_{\text {coll }}(x)$ as

$$
\tau_{\text {coll }}(x)=\tau_{\text {IMVS }}(x) \frac{x}{L-x}
$$

Eq. 6 shows the coupling between the electron transport and recombination processes. According to this equation, as $x \rightarrow L$, the collection characteristic time constant becomes infinite which 
means that the electron cannot be transferred to the external circuit and eventually will be recombined. Now, in the scheme of the RS model, Eq.1 will be modified as

$$
\eta_{\text {coll }}=\frac{\int_{0}^{w} G(x) \eta_{\text {coll }}(x) d x}{\int_{0}^{d} G(x) d x}
$$

Since $\eta_{\text {local }}(x=L)=0$ was assumed in the formulation of the local collection efficiency, the upper limit of the integral has the following values

$$
w= \begin{cases}L, & L \leq d \\ d, & d \leq L\end{cases}
$$

On the other hand, the characteristic time constant of collection is given by [11]

$$
\tau_{\text {coll }}=\frac{\int_{0}^{w} G(x) \eta_{\text {local }}(x) \tau_{\text {coll }}(x) d x}{\int_{0}^{w} G(x) \eta_{\text {local }}(x) d x}
$$

By substituting Eq.4 and Eq.6 into Eq.9 and using definition of Eq.7, we obtain the generalized form of the total collection efficiency as

$$
\frac{\tau_{I M V S}}{\tau_{\text {coll }}}=\frac{\eta_{\text {coll }}}{\frac{\int_{0}^{w} G(x) d x}{\int_{0}^{d} G(x) d x}-\eta_{\text {coll }}}
$$

In the derivation of this equation, the lifetime is assumed to be constant.[11] Obviously, this equation will be reduced to its conventional form of Eq.2 when $w=d(L>d)$. This is a typical condition for high-performance DSSCs, so it can be concluded that Eq. 2 is applicable only for high performance cells in consistency with experimental results [28, 29, 35]. On the other hand, the conditions of $L<d$ which is observed in low-performance DSSCs is also covered by Eq.10. Therefore, this equation can be used to interpret the results of the measurements for all solar cells independent of their specific performance.

As mentioned before, the characteristic IMPS time constant, $\tau_{I M P S}$, in the simplest case is related to $\tau_{\text {coll }}$ and $\tau_{I M V S}$ by $\tau_{I M P S}^{-1}=\tau_{I M V S}^{-1}+\tau_{\text {coll }}^{-1}$ [28]. By introducing this equation into Eq.10, the modified form of the well-known equation $\eta_{\text {coll }}=1-\tau_{I M P S} / \tau_{I M V S}$ is obtained as $\eta_{\text {coll }}=$ $\xi\left(1-\tau_{I M P S} / \tau_{I M V S}\right)$ where $\xi$ is modification factor given by $\xi=\int_{0}^{w} G(x) d x / \int_{0}^{d} G(x) d x$. 


\section{Results and discussion}

\subsection{Comparison with the experimental results}

After combining Eq.9 with Eq.10 and using the definition of the total collection efficiency introduced by Eq.7, we obtain the following equation

$$
\frac{\tau_{I M V S}}{\tau_{I M P S}}=\frac{\int_{0}^{w} G(x) d x}{\int_{0}^{w} G(x) \frac{x}{L} d x}
$$

By utilizing this equation into the Eq.10, we can draw a useful comparison between Eq.2 and its generalized form (Eq.10). Fig.2 shows the comparison between Eq.2 (denoted by $\tilde{\eta}_{\text {coll }}$ ) and Eq.10. For a given absorption coefficient, the diffusion length is swept from $L=0 \mu \mathrm{m}$ to $L=60 \mu \mathrm{m}$, and the network thickness is kept constant at $d=10 \mu \mathrm{m}$. As shown in this figure, the results obtained by the conventional equation (Eq.2) tend towards the results of Eq.10 at high total collection efficiencies. However, there are differences between the two results as the collection efficiency decreases. Such differences are dramatic for the higher absorption coefficients. In a related work, such a comparison is carried out by Sivaram and colleagues [35] based on the experimental data and numerical simulations. The results shown in the Fig.2 are in good agreement with the data reported by Sivaram et al. 


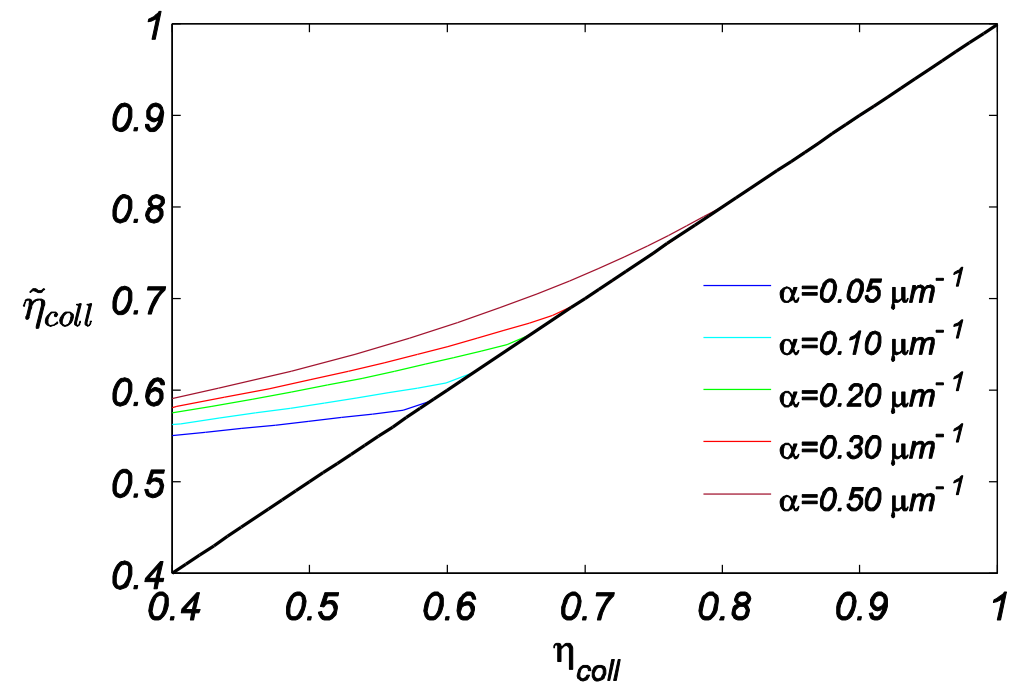

Figure.2 Total collection efficiency for different absorption coefficients obtained by Eq.2 $\left(\tilde{\eta}_{\text {coll }}\right)$ vs. total collection efficiency obtained by Eq.10. The solid black line denotes agreement between Eq. 2 and results of Eq.10. The rate of electron generation, $G(x)$, is given by Eq.13.

\subsection{Comparison with the diffusion equation}

Electronic transport in the DSSCs is governed by diffusion equation $[12,21]$

$$
\frac{\partial n(x, t)}{\partial t}-D \frac{\partial^{2} n(x, t)}{\partial x^{2}}=G(x, t)-k_{r}\left(n^{\beta}(x, t)-n_{b}^{\beta}\right)
$$

where $n(x, t)$ and $n_{b}$ are excess and background electron density, respectively. $\beta$ is the reaction order, $D$ is the electron diffusion coefficient, and $k_{r}$ is the recombination rate constant with dimension of $\left(c m^{3(\beta-1)} / s\right)$. Diffusion length is defined as $L=\sqrt{D(\bar{n})^{1-\beta} / \beta k_{r}}$ where $\overline{\mathrm{n}}=$ $1 / d * \int_{0}^{\mathrm{d}} \mathrm{n}(\mathrm{x}) \mathrm{dx}$ is the mean concentration of electrons in the cell.[21] There is no analytical solution for the diffusion equation in the non-linear recombination model $(\beta \neq 1)$, and it must be solved by numerical approaches. However, the diffusion equation can be solved in the linear recombination $(\beta=1)$ and it was shown that diffusion lengths obtained through linear recombination $(L=\sqrt{D \tau})$ yields physically meaningful values which are approximately equal to the diffusion length predicted by nonlinear recombination. (See ref 14 and appendix B). By using 
Lambert-Beer law, differential generation rate for Substrate Electrode (SE) and Counter Electrode (CE) side illumination is given by

$$
\begin{gathered}
G^{S E}(x) d x=\eta_{i n j} \phi \alpha e^{-\alpha x} d x \\
G^{C E}(x) d x=\eta_{i n j} \phi \alpha e^{-\alpha d} e^{\alpha x} d x
\end{gathered}
$$

where $\alpha$ and $\phi$ are absorption coefficient and incident photon flux, respectively. $\eta_{i n j}$ is the electron injection efficiency. By solving Eq.12, short cicuit current density can be obtained by Fick's law as $J_{s c}=-e D \partial n /\left.\partial x\right|_{x=0}$. On the other hand, Incident Photon Conversion Efficiency (IPCE) at short cicuit is given by

$$
\eta_{I P C E}=\frac{J_{S C}}{q \phi}=\bar{\eta}_{a b s} \bar{\eta}_{i n j} \bar{\eta}_{c o l l}
$$

where $\bar{\eta}_{a b s}, \bar{\eta}_{i n j}$, and $\bar{\eta}_{c o l l}$ are average values of partial quantum efficiencies of light absorption, electron injection, and electron collection, respectively. Assuming $\bar{\eta}_{i n j}=1$, the average of total collection efficiency for SE illumination (Eq.13) can be written as

$$
\bar{\eta}_{\text {coll }}=\frac{J_{s c}}{q \phi\left(1-e^{-\alpha d}\right)}
$$

Fig.3 presents the total collection efficiency as function of $L / d$ calculated from RS model (equations 10 and 11) and numerical solution of diffusion equation (Eq.16). As shown in this figure, the RS model can reproduce the results of the non-linear diffusion equation with a small deviation. This deviation originates from the fact that the RS model ignores the reflection of electrons from the boundary of network (i.e. $\partial n(x) /\left.\partial x\right|_{x=d}=0$ ) which leads to a linear approximation of local collection efficiency. The RS model approaches the exact results as network thickness increases in SE illumination (see SPI.1 for more comparisons). It should be noted that Eq. 2 is in weak agreement with the results of diffusion equation for $L<d$. This argument complements the results of section 3.2. According to Fig.3, it can be concluded that the generalized form of total collection equation (Eq.10) is in good agreement with the diffusion equation and can be used to explain the results of dynamical IMPS and IMVS measurements. 


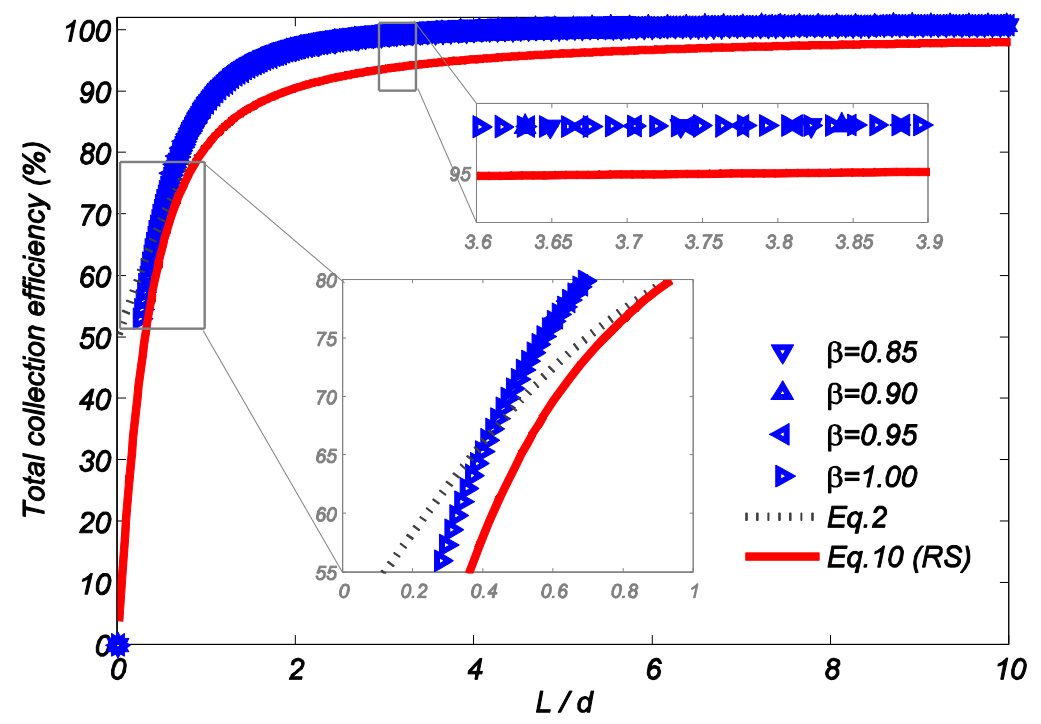

Figure.3 Total collection efficiency as a function of $L / d$ at steady state for SE illumination calculated for $d=10 \mu \mathrm{m}, k_{r}=10^{6} \mathrm{~cm}^{3(\beta-1)} \mathrm{s}^{-1}, n_{b}=10^{15} \mathrm{~cm}^{-3,}, \alpha=0.3 \mu \mathrm{m}^{-1}, \phi=5 \times 10^{16} \mathrm{~cm}^{-2} \mathrm{~s}^{-1}$. Triangle marks represent total collection efficiency obtained by Eq.16 for different $\beta$ values. Solutions giving $n(x)$ and satisfying Eq.12 were computed by MATLAB numerical solver ode15s with the shooting method. Diffusion length is calculated from $=\sqrt{D(\bar{n})^{1-\beta} / \beta k_{r}}$.

\subsection{Direct determination of the electrons diffusion length}

As demonstrated in the Fig.3 and SPI.2, total collection efficiency of DSSCs approaches to its maximum value for $L \geq 2 d$. Eq.11 enables us to explore directly the relationship between time constants and electron diffusion length. By substituting Eq.13 (Eq.14) into Eq.11, we obtain the following results for SE (CE) illumination

$$
\begin{aligned}
& (S E) \frac{\tau_{I M P S}}{\tau_{I M V S}}=\frac{1}{\alpha L}\left(\frac{e^{\alpha w}-\alpha w-1}{e^{\alpha w}-1}\right) \\
& (C E) \frac{\tau_{I M P S}}{\tau_{I M V S}}=\frac{1}{\alpha L}\left(\frac{e^{-\alpha w}+\alpha w-1}{1-e^{-\alpha w}}\right)
\end{aligned}
$$

According to these equations, the relative value of characteristic IMPS and IMVS time constants essentially depends on the relative values of cell thickness and diffusion length. In the case of local profile of photogenerated electrons $(\alpha w \gg 1)$ at SE illumination, eq.18 can be 
approximated as $\tau_{I M P S} / \tau_{I M V S} \approx 1 / \alpha L$. This result provides a direct approach to estimate the electrons diffusion length as a function of mean electron concentration or light intensity.

\section{Conclusion}

A geometrical model was introduced for calculating the electrons local collection efficiency. Using the recombination sphere model, an analytical generalization of the total collection efficiency is obtained as $\eta_{\text {coll }}=\xi\left(1-\tau_{I M P S} / \tau_{I M V S}\right)$ where $\xi$ is modification factor given by $\xi=\int_{0}^{w} G(x) d x / \int_{0}^{d} G(x) d x$. It was shown that this generalized equation is in good agreement with diffusion equation and experimental results. The generalized total collection efficiency obtained in this study can be used to interpret the results of IMPS and IMVS measurements for all solar cells independent of their specific performance. Finally, an approach for direct estimation of the electrons diffusion length was proposed based on the IMPS and IMVS characteristic time constants. Although dye-sensitized solar cells were the subject of this study, analogous arguments based on the recombination sphere model can be applied to the similar solar cells.[40]

\section{Appendix A}

In IMVS measurement, the characteristic recombination time constant can be obtained as follows. Assume that the modulated light of frequency $\omega$ has sufficiently low absorption coefficient which generates a uniform profile within the cell. According to Eq.13, the generation rate is equal to $\eta_{i n j} \alpha \phi e^{i \omega t}$. Since IMVS is measured under the open circuit condition, the electron density is almost constant and one can neglect $\partial^{2} n / \partial x^{2}$ in the diffusion equation. Therefore, Eq.12 reduces to the equation of a highly-dissipated system as (for $\beta=1$ )

$$
\frac{\partial n(\omega, t)}{\partial t}+\frac{n(\omega, t)}{\tau}=G(\omega, t)
$$

Substituting $n(t, \omega)=u(\omega) e^{i \omega t}$ into the above equation leads to 


$$
u(\omega)=\eta_{i n j} \phi \alpha \frac{\tau}{1+i \omega \tau}
$$

The periodic change in the external circuit voltage is proportional to this part of electron density. So

$$
\Delta V(\omega) \propto u(\omega) \propto\left(\eta_{i n j} \phi \alpha \frac{\tau}{1+i \omega \tau}\right)
$$

By separating the real and imaginary parts of the above expression, the $\tau=\tau_{I M V S}=$ $\tau_{\text {rec }}$ obtained as

$$
\tau=-\frac{(\Delta V)_{i m}}{\omega(\Delta V)_{r e}}
$$

\section{Appendix B}

In the linear recombination, by substituting Eq.13 (Eq.14) into Eq.12 and using the short circuit boundary condition as: $\partial n(x) /\left.\partial x\right|_{x=d}=0$ and $n(x=0)=n_{b}$, the photogenerated electrons density can be obtained analytically. By employing Fick's law, we can obtain the short circuit current density as

$$
J_{S C}=\sqrt{\frac{D}{\tau}}\left(Q-n_{b}\right) \tanh \left(\frac{d}{L}\right) \pm Q \alpha D\left(\frac{e^{\mp \alpha d}}{\cosh \left(\frac{d}{L}\right)}-1\right)
$$

The upper (lower) sign refers to the SE (CE) illumination. $L=\sqrt{D \tau}$ is defined as the electrons diffusion length, and $Q$ in the Eq.B1 is different for two sides of illumination as

$$
Q^{S E}=\frac{\eta_{\text {inj }} \alpha I_{0} D}{1-(\alpha L)^{2}}, Q^{C E}=Q^{S E} e^{-\alpha d}
$$

IPCE for two sides of illumination can be achieved by substituting Eq.B1 into Eq.15. In the steady-state measurement, diffusion length and electron injection efficiency are obtained by fitting the experimental results of $I P C E^{C E} / I P C E^{S E}$ to the theoretical prediction of diffusion equation. However, according to the theoretical results (Fig.B1), the corresponding plots of different diffusion lengths begin to overlap for $L>d$. Considering this overlap makes it difficult 
to have an appropriate estimation of diffusion length at aforementioned conditions. This limits the accuracy of steady state measurement to the middle/low-performance cells.[35]

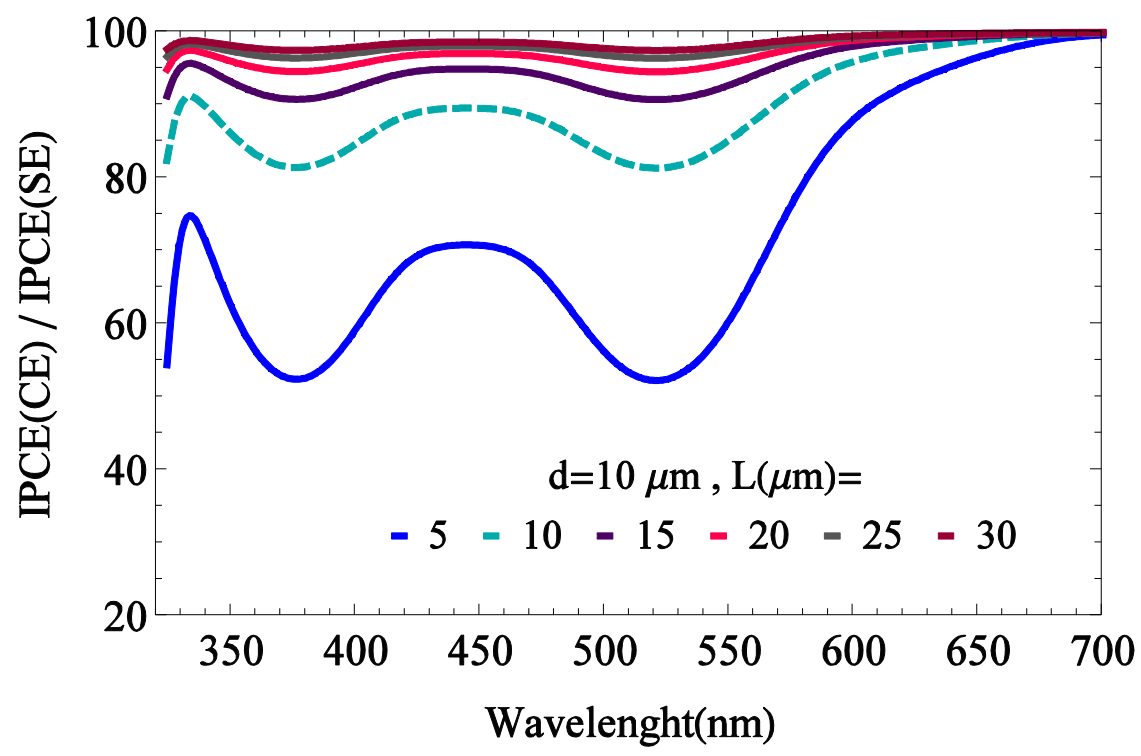

Figure.A1 The relative value of IPCE ${ }^{\mathrm{CE}} / \mathrm{IPCE}^{\mathrm{SE}}$ for a cell with $10 \mu \mathrm{m}$ thickness loaded by simulated N719 sensitizer profile.

\section{Acknowledgements}

We would like to thank the Iran National Science Foundation (INSF) for partial financial support. Partial financial support of the "Centre of Excellence on the Structure of Matter" of the University of Tehran is also acknowledged.

\section{References}

[1] B. O'Regan, M. Grätzel, A low-cost, high-efficiency solar cell based on dyesensitized colloidal TiO2 films, nature, 353 (1991) 737-740.

[2] Y. Bai, I. Mora-Seró, F. De Angelis, J. Bisquert, P. Wang, Titanium dioxide nanomaterials for photovoltaic applications, Chemical reviews, 114 (2014) 1009510130. 
[3] Q. Zhang, C.S. Dandeneau, X. Zhou, G. Cao, ZnO Nanostructures for Dye-Sensitized Solar Cells, Advanced Materials, 21 (2009) 4087-4108.

[4] H. Zheng, Y. Tachibana, K. Kalantar-zadeh, Dye-sensitized solar cells based on WO3, Langmuir, 26 (2010) 19148-19152.

[5] A. Hagfeldt, G. Boschloo, L. Sun, L. Kloo, H. Pettersson, Dye-sensitized solar cells, Chemical reviews, 110 (2010) 6595-6663.

[6] S. Rühle, M. Shalom, A. Zaban, Quantum-dot-sensitized solar cells, ChemPhysChem, 11 (2010) 2290-2304.

[7] M.R. Kim, D. Ma, Quantum-Dot-Based Solar Cells: Recent Advances, Strategies, and Challenges, The Journal of Physical Chemistry Letters, 6 (2014) 85-99.

[8] J. Burschka, N. Pellet, S.-J. Moon, R. Humphry-Baker, P. Gao, M.K. Nazeeruddin, M. Grätzel, Sequential deposition as a route to high-performance perovskite-sensitized solar cells, Nature, 499 (2013) 316-319.

[9] S. Nakade, T. Kanzaki, W. Kubo, T. Kitamura, Y. Wada, S. Yanagida, Role of electrolytes on charge recombination in dye-sensitized tio2 solar cell (1): the case of solar cells using the I-/I3-redox couple, The Journal of Physical Chemistry B, 109 (2005) 3480-3487.

[10] I. Chung, B. Lee, J. He, R.P. Chang, M.G. Kanatzidis, All-solid-state dyesensitized solar cells with high efficiency, Nature, 485 (2012) 486-489.

[11] J. Van de Lagemaat, N.-G. Park, A. Frank, Influence of electrical potential distribution, charge transport, and recombination on the photopotential and photocurrent conversion efficiency of dye-sensitized nanocrystalline TiO2 solar cells: a study by electrical impedance and optical modulation techniques, The Journal of Physical Chemistry B, 104 (2000) 2044-2052.

[12] J. Bisquert, I. Mora-Seró, Simulation of steady-state characteristics of dyesensitized solar cells and the interpretation of the diffusion length, The Journal of Physical Chemistry Letters, 1 (2009) 450-456.

[13] K. Zhu, N.R. Neale, A. Miedaner, A.J. Frank, Enhanced charge-collection efficiencies and light scattering in dye-sensitized solar cells using oriented $\mathrm{TiO} 2$ nanotubes arrays, Nano letters, 7 (2007) 69-74.

[14] P.R. Barnes, L. Liu, X. Li, A.Y. Anderson, H. Kisserwan, T.H. Ghaddar, J.R. Durrant, B.C. O'Regan, Re-evaluation of recombination losses in dye-sensitized cells: the failure of dynamic relaxation methods to correctly predict diffusion length in nanoporous photoelectrodes, Nano letters, 9 (2009) 3532-3538. 
[15] A.B. Martinson, M.S. Góes, F. Fabregat-Santiago, J. Bisquert, M.J. Pellin, J.T. Hupp, Electron transport in dye-sensitized solar cells based on $\mathrm{ZnO}$ nanotubes: evidence for highly efficient charge collection and exceptionally rapid dynamicst, The Journal of Physical Chemistry A, 113 (2009) 4015-4021.

[16] E. Ghadiri, N. Taghavinia, S.M. Zakeeruddin, M. Grätzel, J.-E. Moser, Enhanced electron collection efficiency in dye-sensitized solar cells based on nanostructured TiO2 hollow fibers, Nano letters, 10 (2010) 1632-1638.

[17] H. Yang, G.H. Guai, C. Guo, Q. Song, S.P. Jiang, Y. Wang, W. Zhang, C.M. Li, $\mathrm{NiO} / g$ raphene composite for enhanced charge separation and collection in p-type dye sensitized solar cell, The Journal of Physical Chemistry C, 115 (2011) 1220912215.

[18] J.P. Gonzalez-Vazquez, G. Bigeriego, J.A. Anta, Influence of the charge generation profile on the collection efficiency of nanostructured solar cells: a random walk numerical simulation study, Molecular Simulation, 38 (2012) 12421250.

[19] P.R. Barnes, A.Y. Anderson, S.E. Koops, J.R. Durrant, B.C. O’Regan, Electron injection efficiency and diffusion length in dye-sensitized solar cells derived from incident photon conversion efficiency measurements, The Journal of Physical Chemistry C, 113 (2008) 1126-1136.

[20] J. Halme, G. Boschloo, A. Hagfeldt, P. Lund, Spectral characteristics of light harvesting, electron injection, and steady-state charge collection in pressed TiO2 dye solar cells, The Journal of Physical Chemistry C, 112 (2008) 5623-5637.

[21] P.R. Barnes, B.C. O'Regan, Electron recombination kinetics and the analysis of collection efficiency and diffusion length measurements in dye sensitized solar cells, The Journal of Physical Chemistry C, 114 (2010) 19134-19140.

[22] S. Nakade, Y. Saito, W. Kubo, T. Kanzaki, T. Kitamura, Y. Wada, S. Yanagida, Laser-induced photovoltage transient studies on nanoporous TiO2 electrodes, The Journal of Physical Chemistry B, 108 (2004) 1628-1633.

[23] B.C. O'Regan, F. Lenzmann, Charge transport and recombination in a nanoscale interpenetrating network of $n$-type and $p$-type semiconductors: transient photocurrent and photovoltage studies of TiO2/Dye/CuSCN photovoltaic cells, The Journal of Physical Chemistry B, 108 (2004) 4342-4350.

[24] S. Nakade, T. Kanzaki, Y. Wada, S. Yanagida, Stepped light-induced transient measurements of photocurrent and voltage in dye-sensitized solar cells: 
application for highly viscous electrolyte systems, Langmuir, 21 (2005) 1080310807.

[25] P.R. Barnes, K. Miettunen, X. Li, A.Y. Anderson, T. Bessho, M. Gratzel, B.C. O'Regan, Interpretation of Optoelectronic Transient and Charge Extraction Measurements in Dye-Sensitized Solar Cells, Advanced materials, 25 (2013) 18811922.

[26] L. Dloczik, O. Ileperuma, I. Lauermann, L. Peter, E. Ponomarev, G. Redmond, N. Shaw, I. Uhlendorf, Dynamic response of dye-sensitized nanocrystalline solar cells: characterization by intensity-modulated photocurrent spectroscopy, The Journal of Physical Chemistry B, 101 (1997) 10281-10289.

[27] G. Schlichthörl, S. Huang, J. Sprague, A. Frank, Band edge movement and recombination kinetics in dye-sensitized nanocrystalline TiO2 solar cells: a study by intensity modulated photovoltage spectroscopy, The Journal of Physical Chemistry B, 101 (1997) 8141-8155.

[28] G. Schlichthörl, N. Park, A. Frank, Evaluation of the charge-collection efficiency of dye-sensitized nanocrystalline TiO2 solar cells, The Journal of Physical Chemistry B, 103 (1999) 782-791.

[29] J. Halme, K. Miettunen, P. Lund, Effect of nonuniform generation and inefficient collection of electrons on the dynamic photocurrent and photovoltage response of nanostructured photoelectrodes, The Journal of Physical Chemistry C, 112 (2008) 20491-20504.

[30] J. Bisquert, Theory of the impedance of electron diffusion and recombination in a thin layer, The Journal of Physical Chemistry B, 106 (2002) 325-333.

[31] F. Fabregat-Santiago, J. Bisquert, G. Garcia-Belmonte, G. Boschloo, A. Hagfeldt, Influence of electrolyte in transport and recombination in dye-sensitized solar cells studied by impedance spectroscopy, Solar Energy Materials and Solar Cells, 87 (2005) 117-131.

[32] J. Bisquert, L. Bertoluzzi, I. Mora-Sero, G. Garcia-Belmonte, Theory of impedance and capacitance spectroscopy of solar cells with dielectric relaxation, drift-diffusion transport, and recombination, The Journal of Physical Chemistry C, 118 (2014) 18983-18991.

[33] P. De Jongh, D. Vanmaekelbergh, Investigation of the electronic transport properties of nanocrystalline particulate $\mathrm{TiO} 2$ electrodes by intensity-modulated 
photocurrent spectroscopy, The Journal of Physical Chemistry B, 101 (1997) 27162722.

[34] J. Krüger, R. Plass, M. Grätzel, P.J. Cameron, L.M. Peter, Charge transport and back reaction in solid-state dye-sensitized solar cells: a study using intensitymodulated photovoltage and photocurrent spectroscopy, The Journal of Physical Chemistry B, 107 (2003) 7536-7539.

[35] V. Sivaram, J. Kirkpatrick, H. Snaith, Critique of charge collection efficiencies calculated through small perturbation measurements of dye sensitized solar cells, Journal of Applied Physics, 113 (2013) 063709.

[36] L. Bertoluzzi, S. Ma, On the methods of calculation of the charge collection efficiency of dye sensitized solar cells, Physical Chemistry Chemical Physics, 15 (2013) 4283-4285.

[37] J. van de Lagemaat, N.G. Park, A.J. Frank, Influence of Electrical Potential Distribution, Charge Transport, and Recombination on the Photopotential and Photocurrent Conversion Efficiency of Dye-Sensitized Nanocrystalline TiO2Solar Cells: A Study by Electrical Impedance and Optical Modulation Techniques, The Journal of Physical Chemistry B, 104 (2000) 2044-2052.

[38] J. Halme, G. Boschloo, A. Hagfeldt, P. Lund, Spectral Characteristics of Light Harvesting, Electron Injection, and Steady-State Charge Collection in Pressed TiO2Dye Solar Cells, The Journal of Physical Chemistry C, 112 (2008) 5623-5637.

[39] J.R. Jennings, F. Li, Q. Wang, Reliable determination of electron diffusion length and charge separation efficiency in dye-sensitized solar cells, The Journal of Physical Chemistry C, 114 (2010) 14665-14674.

[40] T. Kirchartz, J. Bisquert, I. Mora-Sero, G. Garcia-Belmonte, Classification of solar cells according to mechanisms of charge separation and charge collection, Physical Chemistry Chemical Physics, 17 (2015) 4007-4014. 